\title{
Transcriptome sequencing reveals dynamic changes in matrix metalloproteinases in facet joint osteoarthritis
}

\author{
CHU CHEN*, GUANHUA XU*, YUYU SUN and ZHIMING CUI \\ Department of Spine Surgery, The Second Affiliated Hospital of Nantong University, Nantong, Jiangsu 226001, P.R. China
}

Received July 9, 2019; Accepted December 4, 2019

DOI: $10.3892 /$ etm.2020.8488

\begin{abstract}
Osteoarthritis is a general joint disease characterized by articular cartilage degeneration. The extracellular matrix is a principal component in articular cartilage. The dynamic remodeling of the extracellular matrix is involved in the pathological degradation of the articular cartilage. Facet joint osteoarthritis (FJOA) is a common form of osteoarthritis that occurs in the posterior aspect of the vertebral column. However, to the best of our knowledge, the current understanding of the genetic changes in FJOA is limited. The most significantly differentially expressed genes and Gene Ontology categories in FJOA were identified by transcriptome sequencing analysis. The extracellular matrix, matrix metalloproteinases (MMPs) and proteinases of the extracellular matrix were highly involved in FJOA. The canonical signaling pathway 'inhibition of matrix metalloproteinases' was further studied in detail by identifying and validating differentially expressed genes in the signaling pathway. Taken together, the present study revealed changes in MMP-related genes in FJOA and showed the importance of extracellular matrix remodeling in FJOA from a genetic aspect.
\end{abstract}

\section{Introduction}

Osteoarthritis is a common joint disease mainly characterized by the degeneration and breakdown of articular cartilage, the formation of osteophytes and subchondral cysts, and the thickening of subchondral bone (1). Articular cartilage is a resilient and smooth tissue that covers and protects the ends of bones in joints, and primarily consists of a dense extracellular matrix with no blood vessels, lymphatic vessels or nerves, with a sparse chondrocyte population $(2,3)$.

Correspondence to: Dr Zhiming Cui, Department of Spine Surgery, The Second Affiliated Hospital of Nantong University, 6 Hai'er Alley North, Nantong, Jiangsu 226001, P.R. China

E-mail: czmspine@126.com

${ }^{*}$ Contributed equally

Key words: facet joint osteoarthritis, transcriptome sequencing, bioinformatics analysis, extracellular matrix, matrix metalloproteinases
The extracellular matrix is a multifaceted meshwork of proteoglycans, polysaccharides and proteins secreted by resident supporting cells. The extracellular matrix not only represents a critical structural component of articular cartilage, but is also involved in the pathological degradation of articular cartilage (4-6). Physical and biochemical alterations in the extracellular matrix may elicit numerous signaling events by communicating with the intracellular cytoskeleton, stimulating chondrocyte responses and thus inducing osteoarthritis (7-9). The dynamic remodeling and homeostasis of the extracellular matrix is regulated by a variety of proteinases, especially proteinases in the matrix metalloproteinase (MMP) family $(10,11)$.

Facet joint osteoarthritis (FJOA) is a common form of osteoarthritis that occurs in the facet joints located in the posterior aspect of the vertebral column (12). However, to the best of our knowledge, FJOA is not as well studied as other forms of osteoarthritis, such as hip osteoarthritis and knee osteoarthritis. Efforts have been made to examine the anatomy, biomechanics, epidemiology and clinical manifestations of FJOA (13). The expression of key molecules in the degenerative process of facet joints such as MMP13, tumor necrosis factor-a and interleukin- 6 have also been identified (14). A previous study determined the molecular changes in FJOA by collecting human facet joint tissues from healthy controls and patients with FJOA and conducting transcriptome sequencing (15).

The current study investigated the expression profiles of genes in healthy controls and patients with FJOA by filtering genes with the most significant fold-changes and screening the most enriched Gene Ontology (GO) categories. Analysis results showed that MMP12, a member of the MMP family, was the most downregulated gene in FJOA. In addition, many enriched GO categories were related to the extracellular matrix. Therefore, the canonical signaling pathway 'inhibition of matrix metalloproteinases' was investigated through the joint use of Ingenuity Pathway Analysis (IPA) software, heatmap and hierarchical cluster analysis, and reverse transcription-quantitative PCR (RT-qPCR) validation to study the involvement of MMPs in FJOA.

\section{Materials and methods}

Facet joint tissue collection and transcriptome sequencing. Human facet joint tissues were collected from 48 patients 
with FJOA (FJOA group) and 10 healthy patients with vertebral fractures (control group) as previously described (15). Magnetic resonance imaging was applied to determine the degree of cartilage degeneration. The facet joint tissues of patients in the FJOA group were of Grade 2 (moderate degeneration) and Grade 3 (severe degeneration), while the facet joint tissue of patients in the control group was of Grade 0 (normal) and Grade 1 (mild degeneration) (16). Patients with infection, inflammatory diseases or autoimmune diseases were excluded from the current study. The present study was ethically approved by the Human Ethics Committee of the Second Affiliated Hospital of Nantong University. All participants provided written informed consent.

Facet joint tissues from the FJOA and control groups were divided into three parts for technical repetitions for subsequent RNA isolation and sequencing experiments. Total RNA was extracted using TRIzol ${ }^{\circledR}$ reagent (Invitrogen; Thermo Fisher Scientific, Inc.) according to the manufacturer's instructions. Extracted total RNA was used to fragment RNA and synthesize cDNA. A cDNA library was amplified and sequenced using a Hiseq X Ten sequencing platform (Illumina, Inc.) to produce raw reads. Dirty reads were filtered to obtain clean reads. Clean reads were then mapped to the reference genome using TopHat v2.1.1 (https://ccb.jhu.edu/software/tophat/index. shtml) $(17,18)$.

Bioinformatics analysis. Transcriptome sequencing data were used to identify the fragments per kilobase of transcript per million mapped reads (FPKM) values of each gene. The FPKM values of genes in the FJOA group were compared with the FPKM values in the control group to screen differentially expressed genes with a fold-change $>2$ or $<-2\left(\log _{2}\right.$ ratio $>1$ or $<-1$ ) and a q-value (adjusted P-value) $<0.05$. The Database for Annotation, Visualization, and Integrated Discovery (DAVID) 6.7 (https://david.ncifcrf.gov/) was used to enrich differentially expressed genes to GO categories (19-21). IPA software (2018 release Qiagen, Inc.) was used to analyze the canonical signaling pathway 'inhibition of matrix metalloproteinases'. Heatmap and hierarchical cluster analyses were performed using R v2.13.0 (https://www.r-project.org/) to display the expression patterns of differentially expressed genes.

$R T-q P C R$. RT-qPCR was conducted to validate transcriptome sequencing outcomes. RNA samples were first reverse transcribed to cDNA using the PrimeScript RT Reagent kit (Takara Biotechnology Co., Ltd.) according to the manufacturer's protocol. qPCR was performed using SYBR Premix Ex Taq (Takara Biotechnology Co., Ltd) on a StepOnePlus Real-Time PCR system (Applied Biosystems; Thermo Fisher Scientific, Inc.). The following thermocycling conditions were used for the PCR: Initial denaturation at $95^{\circ} \mathrm{C}$ for $5 \mathrm{~min} ; 40$ cycles of $95^{\circ} \mathrm{C}$ at $30 \mathrm{sec}, 45 \mathrm{sec}$ at $56.5^{\circ} \mathrm{C}$ and $72^{\circ} \mathrm{C}$ for $30 \mathrm{sec}$; and a final extension at $72^{\circ} \mathrm{C}$ for $5 \mathrm{~min}$. The relative expression of target genes MMP7, disintegrin and metalloproteinase domain-containing protein 12 (ADAM12), tissue inhibitor of metalloproteinases 3 (TIMP3) and TIMP4 were calculated using the $2^{-\Delta \Delta C q}$ method with GAPDH as the internal reference gene (22). The sequences of primers pairs used in the qPCR are listed in Table I.
Table I. Primer pairs for reverse transcription-quantitative PCR.

\begin{tabular}{lll}
\hline Gene & Primer & \multicolumn{1}{c}{ Sequence (5'-3') } \\
\hline MMP7 & $\begin{array}{l}\text { Forward } \\
\text { AGCAGCTATGCAGCTGGCCGT }\end{array}$ & AGC \\
ADAM12 & Forward & TCCCTGACTGCCTGTTCCCACTGC \\
& Reverse & AAGGGCGCACACACCTTAGTTT \\
TIMP3 & Forward & AGGACGCCTTCTGCAAC \\
& Reverse & CTCCTTTACCAGCTTCTTCC \\
TIMP4 & Forward & ACCTGTCCTTGGTGCAGA \\
& Reverse & TGTAGCAGGTGGTGATTGG \\
GAPDH & Forward & CCAAGGTCATCCATGACAAC \\
& Reverse & TGTCATACCAGGAAATGAGC
\end{tabular}

MMP7, matrix metalloproteinase 7; ADAM12, disintegrin and metalloproteinase domain-containing protein 12; TIMP, tissue inhibitor of metalloproteinases.

Statistical analysis. Data are presented as the mean \pm SEM and were analyzed using GraphPad Prism 5.0 (GraphPad Software, Inc.). Student's t-test was used to compare differences between the FJOA and control groups. $\mathrm{P}<0.05$ was considered to indicate a statistically significant difference.

\section{Results}

Identification of the most differentially expressed genes in $F J O A$. Previous transcriptome sequencing determined the expression levels of $>19,000$ genes in human facet joint tissues in both FJOA and control groups (15). To obtain a global view of the expression patterns of identified genes, the Heatmap function in the software platform R (v.2.13.0) was used to draw a heatmap of gene expression (Fig. 1). The Euclidean distance was calculated using the Hierarchical Clustering module from GenePattern (https://software.broadinstitute.org/cancer/software/genepattern/) to cluster the identified genes (Fig. 1) (23). Parallelizing hierarchical clustering methods were used to study the gene expression profiles in the three control group replicates (CTRL1, CTRL2 and CTRL3) and three FJOA group replicates (FJOA1, FJOA2 and FJOA3) (Fig. 1). Compared with genes in the control group, a smaller number of genes in the FJOA group were upregulated while a relatively large number of genes were downregulated.

A detailed investigation of these differentially expressed genes showed that the expression level of the most upregulated gene in FJOA, corticotropin releasing hormone receptor 1 (CRHR1), was elevated $>28.8$ fold $\left(\log _{2}\right.$ ratio $\left.=4.85\right)$ compared with the control group. The expression level of the most downregulated gene, MMP12, was reduced to $\sim 1 / 14$ of its expression level compared with the control group $\left(\log _{2}\right.$ ratio=-3.86). The 10 most upregulated and downregulated genes in FJOA are listed in Table II.

Identification of most enriched GO categories in FJOA. To have a better understanding of the molecular mechanisms underlying FJOA, the DAVID database was used to group 
Table II. Top 10 upregulated and downregulated genes in FJOA.

A, Upregulated

\begin{tabular}{lccccc}
\hline GeneID & CTRL_FPKM & FJOA_FPKM & $\log _{2}($ FJOA/CTRL $)$ & q-value & Description \\
\hline CRHR1 & 0.022303 & 0.643232 & 4.85003 & 0.00463 & $\begin{array}{l}\text { Corticotropin releasing hormone } \\
\text { receptor 1 }\end{array}$ \\
CSF3 & 0.230299 & 3.21825 & 3.80469 & 0.000259 & Colony stimulating factor 3 \\
CRLF1 & 6.24624 & 81.3432 & 3.70296 & 0.000259 & Cytokine receptor like factor 1 \\
DAW1 & 0.090234 & 1.12819 & 3.6442 & 0.022496 & $\begin{array}{l}\text { Dynein assembly factor with } \\
\text { WD repeats 1 }\end{array}$ \\
HAS1 & 2.90011 & 35.0671 & 3.59594 & 0.000259 & Hyaluronan synthase 1 \\
POSTN & 23.7872 & 265.481 & 3.48035 & 0.000259 & Periostin \\
TMEM59L & 0.181724 & 1.78416 & 3.29542 & 0.005947 & Transmembrane protein 59 like \\
PVRL4 & 0.170113 & 1.61362 & 3.24574 & 0.000259 & Poliovirus receptor-related \\
protein 4 & Long intergenic non-protein \\
LINC00702 & 0.924058 & 8.50754 & 3.20269 & 0.002128 & coding RNA 702 \\
HMCN2 & 0.158359 & 1.36891 & 3.11176 & 0.000259 & Hemicentin 2 \\
\hline
\end{tabular}

B, Downregulated

\begin{tabular}{lccccc}
\hline GeneID & CTRL_FPKM & FJOA_FPKM & $\log _{2}($ FJOA/CTRL) & q-value & Description \\
\hline MMP12 & 1.5777 & 0.10857 & -3.86112 & 0.013616 & Matrix metallopeptidase 12 \\
HBG1 & 57.9551 & 4.16107 & -3.79991 & 0.000259 & Hemoglobin subunit $\gamma 1$ \\
SPIC & 2.63717 & 0.231806 & -3.508 & 0.00394 & Spi-c transcription factor \\
TPSB2 & 8.40967 & 0.772965 & -3.44357 & 0.000259 & Tryptase $\beta 2$ (gene/pseudogene) \\
SEC14L3 & 0.596955 & 0.059692 & -3.32201 & 0.007555 & Sec14 like lipid binding 3 \\
PYHIN1 & 2.77976 & 0.280398 & -3.30942 & 0.000259 & Pyrin and hin domain family \\
& & & & & member 1 \\
CHI3L1 & 1078.35 & 114.902 & -3.23036 & 0.000259 & Chitinase 3 like 1 \\
CD19 & 1.2214 & 0.131175 & -3.21897 & 0.004793 & Cd19 molecule \\
GP5 & 3.14407 & 0.360364 & -3.12511 & 0.000259 & Glycoprotein v platelet \\
GPR15 & 1.43198 & 0.166889 & -3.10106 & 0.033956 & G protein-coupled receptor 15
\end{tabular}

FPKM, fragments per kilobase of transcript per million mapped reads; FJOA, facet joint osteoarthritis; CTRL, control.

differentially expressed genes into GO biological processes, molecular functions and cellular components (Fig. 2). The ten most enriched GO biological processes were associated with the response of the organism ('response to stress' and 'response to stimulus'), immune response ('regulation of immune system process', 'positive regulation of immune system process', 'immune system process', 'immune response' and 'defense response') and cellular behaviors ('cell adhesion', 'cell activation' and 'biological adhesion'). The significant involvement of the immune response was consistent with the high expression of CRHR1 observed in FJOA tissue, as CRHR1 is essential for the activation of various signaling pathways that regulate the immune response. The ten most enriched GO molecular functions were mainly related to molecular binding, while the ten most enriched GO cellular components included various components, especially those involving the extracellular matrix such as 'proteinaceous extracellular matrix', 'extracellular space', 'extracellular region part', 'extracellular region' and 'extracellular matrix'.

Analysis of the canonical signaling pathway 'inhibition of matrix metalloproteinases'. GO cellular component analysis showed that the extracellular matrix was highly associated with FJOA. In addition, MMP12, a gene that encodes for an enzyme involved in the breakdown of the extracellular matrix, was most significantly downregulated in FJOA. The results of the bioinformatics analysis suggested that dysregulated MMPs and the resulting modulated extracellular matrix might be critical in the pathological process of FJOA. Therefore, IPA bioinformatics analysis was performed to identify the involvement of the canonical signaling pathway 'inhibition of matrix metalloproteinases' in FJOA.

The schematic diagram of the canonical signaling pathway 'inhibition of matrix metalloproteinases' is shown in Fig. 3. 
Table III. Expression levels of differentially expressed genes in the canonical signaling pathway 'inhibition of matrix metalloproteinases'.

\begin{tabular}{|c|c|c|c|c|c|}
\hline GeneID & CTRL_FPKM & FJOA_FPKM & $\log _{2}(\mathrm{FJOA} / \mathrm{CTRL})$ & q-value & Description \\
\hline $\mathrm{A} 2 \mathrm{M}$ & 152.6 & 144.685 & -0.07684 & 0.580386 & $\alpha$-2-macroglobulin \\
\hline ADAM10 & 23.1911 & 18.2402 & -0.34645 & 0.00269 & $\begin{array}{l}\text { Disintegrin and metalloproteinase domain- } \\
\text { containing protein } 10\end{array}$ \\
\hline ADAM12 & 5.90052 & 19.1302 & 1.69694 & 0.000259 & $\begin{array}{l}\text { Disintegrin and metalloproteinase domain- } \\
\text { containing protein } 12\end{array}$ \\
\hline ADAM17 & 12.845 & 12.1117 & -0.08481 & 0.581591 & $\begin{array}{l}\text { Disintegrin and metalloproteinase domain- } \\
\text { containing protein } 17\end{array}$ \\
\hline HSPG2 & 79.3027 & 132.242 & 0.737738 & 0.000259 & $\begin{array}{l}\text { Basement membrane-specific heparan sulfate } \\
\text { proteoglycan core protein }\end{array}$ \\
\hline LRP1 & 251.144 & 326.912 & 0.38039 & 0.012286 & $\begin{array}{l}\text { Prolow-density lipoprotein receptor-related } \\
\text { protein } 1\end{array}$ \\
\hline MMP2 & 108.741 & 175.485 & 0.690452 & 0.000259 & Matrix metalloproteinase 2 \\
\hline MMP9 & 314.457 & 91.4777 & -1.78137 & 0.000259 & Matrix metalloproteinase 9 \\
\hline MMP17 & 1.17006 & 1.48701 & 0.345823 & 0.353116 & Matrix metalloproteinase 17 \\
\hline MMP11 & 5.50944 & 8.16829 & 0.568128 & 0.001552 & Matrix metalloproteinase 11 \\
\hline MMP3 & 373.253 & 167.156 & -1.15896 & 0.000259 & Matrix metalloproteinase 3 \\
\hline MMP19 & 6.38089 & 10.853 & 0.766262 & 0.000497 & Matrix metalloproteinase 19 \\
\hline MMP13 & 125.361 & 75.5355 & -0.73086 & 0.000259 & Matrix metalloproteinase 13 \\
\hline MMP28 & 2.48386 & 4.40147 & 0.825399 & 0.026506 & Matrix metalloproteinase 28 \\
\hline MMP23B & 0.04404 & 0.828743 & 4.23405 & 0.247905 & Matrix metalloproteinase 23B \\
\hline MMP14 & 74.0875 & 118.202 & 0.673953 & 0.000259 & Matrix metalloproteinase 14 \\
\hline MMP24 & 1.21904 & 1.34362 & 0.14038 & 0.778023 & Matrix metalloproteinase 24 \\
\hline MMP8 & 37.9945 & 14.832 & -1.35708 & 0.000259 & Matrix metalloproteinase 8 \\
\hline MMP1 & 6.78886 & 4.20773 & -0.69013 & 0.493474 & Matrix metalloproteinase 1 \\
\hline MMP12 & 1.5777 & 0.10857 & -3.86112 & 0.013616 & Matrix metalloproteinase 12 \\
\hline MMP15 & 1.65603 & 2.41486 & 0.54421 & 0.016667 & Matrix metalloproteinase 15 \\
\hline MMP16 & 9.42333 & 4.70063 & -1.00338 & 0.000259 & Matrix metalloproteinase 16 \\
\hline MMP7 & 2.13341 & 1.03658 & -1.04133 & 0.028971 & Matrix metalloproteinase 7 \\
\hline MMP25 & 6.06705 & 2.5246 & -1.26494 & 0.000259 & Matrix metalloproteinase 25 \\
\hline RECK & 9.33448 & 14.4833 & 0.633749 & 0.000259 & $\begin{array}{l}\text { Reversion-inducing cysteine-rich protein with } \\
\text { Kazal motifs }\end{array}$ \\
\hline TFPI2 & 0.973482 & 0.789919 & -0.30145 & 0.53558 & Tissue factor pathway inhibitor 2 \\
\hline TIMP1 & 322.419 & 227.107 & -0.50557 & 0.000259 & Tissue inhibitor of metalloproteinases 1 \\
\hline TIMP2 & 216.411 & 377.86 & 0.804078 & 0.000259 & Tissue inhibitor of metalloproteinases 2 \\
\hline TIMP3 & 91.4082 & 261.077 & 1.51408 & 0.000259 & Tissue inhibitor of metalloproteinases 3 \\
\hline TIMP4 & 27.8848 & 57.9526 & 1.05539 & 0.000259 & Tissue inhibitor of metalloproteinases 4 \\
\hline TSP2 & 37.7629 & 73.7939 & 0.966534 & 0.000259 & Thrombospondin-2 \\
\hline
\end{tabular}

FPKM, fragments per kilobase of transcript per million mapped reads; FJOA, facet joint osteoarthritis; CTRL, control.

MMPs are calcium-dependent zinc-containing endopeptidases that are critical for the modulation of both cell-cell and cell-extracellular matrix interactions (24). These MMPs are tightly regulated at multiple levels, including gene transcription, enzyme activation and inhibitor inactivation (25). TIMPs are a family of protease inhibitors comprising TIMP1, TIMP2, TIMP3 and TIMP4. TIMPs form non-covalent bonds with the latent and active forms of MMPs with a 1:1 stoichiometry and thus inhibit the activity of MMPs (26). Members of the ADAM family, such as ADAM10, ADAM12 and ADAM13 also regulate cell adhesion and mediate the extracellular matrix (27) (Fig. 3).

Upregulated and downregulated genes in the canonical signaling pathway 'inhibition of matrix metalloproteinases' in FJOA are labeled in red and green, respectively (Fig. 3). The expression levels of genes in the pathway are shown in Table III. Heatmap and relevant hierarchical clustering analyses were performed to illustrate the expression levels of genes in the FJOA and control groups (Fig. 4). Most members of the MMP family (including MMP12, MMP9, MMP3, MMP16, 


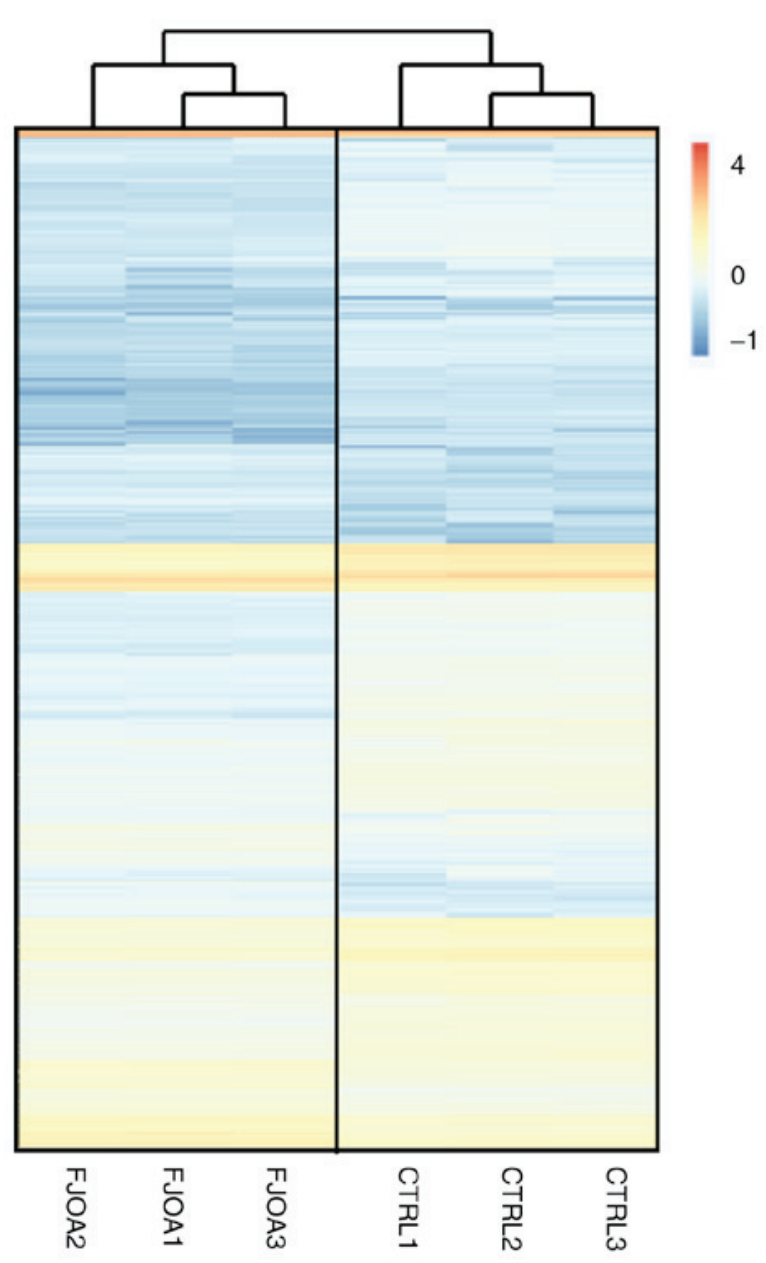

Figure 1. Expression patterns of genes in healthy and lesioned facet joint tissues. The expression levels of genes are shown in a heatmap and indicated by a color bar. Red indicates upregulated genes while blue indicates downregulated genes.

MMP7, MMP8 and MMP25) were significantly downregulated in FJOA compared with controls. Meanwhile, MMP23B was upregulated and the expression levels of other members of the MMP family were not significantly altered compared with controls. ADAM12 and MMP inhibitors TIMP3 and TIMP4 were upregulated in FJOA compared with controls.

Validation using $R T$-qPCR. RT-qPCR experiments were further conducted to measure the expression of genes in FJOA and control groups in order to validate transcriptome sequencing results. Representative genes in the canonical signaling pathway 'inhibition of matrix metalloproteinases', MMP7, ADAM12, TIMP3 and TIMP4 were selected for RT-qPCR experiments (Fig. 5). Consistent with the transcriptome sequencing results, the expression levels of MMP7 were significantly downregulated in the FJOA group compared with the control group. By contrast, the expression levels of ADAM12, TIMP3 and TIMP4 were significantly higher in the FJOA group as compared with the control group (Fig. 5).

\section{Discussion}

In the current study, transcriptome sequencing outcomes from human facet joint tissues in FJOA patients and healthy controls were analyzed. The ten most significantly upregulated and downregulated genes were identified and the ten most enriched GO biological processes, molecular functions and cellular components were categorized. The analytical results showed that the extracellular matrix was robustly involved in the genetic changes involved in FJOA. MMP12, a calcium-dependent zinc-containing endopeptidase capable of degrading the extracellular matrix, was the most downregulated gene in FJOA. CRHR1, which encodes a G-protein coupled receptor that binds neuropeptides of the corticotropin releasing hormone family that are major regulators of the hypothalamic-pituitary-adrenal pathway, was the most differentially expressed gene in FJOA. From a gene expression aspect, the results suggested that modulation of the extracellular matrix is a critical biological process in FJOA, and the CRHR1-regulated immune response might be critical in the development of FJOA. Treatments targeting CRHR1 and MMP might be potential therapeutic methods. Therefore, gene changes in the canonical signaling pathway 'inhibition of matrix metalloproteinases' were examined. Heatmap analysis and RT-qPCR experiments illustrated that several genes in 'inhibition of matrix metalloproteinases', especially MMPs and TIMPs, were dysregulated in FJOA.

The extracellular matrix is a non-cellular meshwork of extracellular molecules that provides a three-dimensional physical and mechanical support for surrounding cells (28). The extracellular matrix is highly dynamic and is continuously remodeled by matrix-degrading enzymes under various physiological and pathological conditions, including cell migration, cell differentiation, cell growth, wound healing and fibrosis (28-31). The remodeling of the extracellular matrix is principally mediated by MMPs $(32,33)$. Changes in the composition and structure of the extracellular matrix, such as loss of proteoglycans, mineralization of the extracellular matrix and accumulation of malformed extracellular matrix are hallmarks of osteoarthritis $(8,34)$. The present study showed that genes coding for many MMPs were downregulated in FJOA. Consistent with sequencing data, MMP7 was significantly downregulated in FJOA compared with controls. Since MMP12 was the most downregulated gene in FJOA, the biological functions of MMP12 can be investigated in future studies. In addition, TIMP3 and TIMP4 were found to be upregulated in FJOA. Elevated TIMPs further inactivate MMPs and influence the dynamics of the extracellular matrix (35).

In contrast with the present observations, many members of the MMP family were upregulated and/or activated in many other forms of osteoarthritis (36-38). The contradictory results implied that the molecular changes in joint tissues in osteoarthritis might be tissue-specific. Kim et al (39) reported that MMP1, MMP3 and MMP13 were upregulated in human degenerative facet joint tissues, however, MMP3 expression was downregulated in the present study. Aside from the differences in the collection of human facet joint tissues and the classification of FJOA, another difference was that the current study focused on the Chinese population while the study performed by Kim et al performed studies on tissues collected from an American population (39). Meta analyses could be conducted to investigate the association between genetic changes in FJOA and different populations. Further studies will be conducted to examine the protein expression and localization of these MMPs in FJOA. 

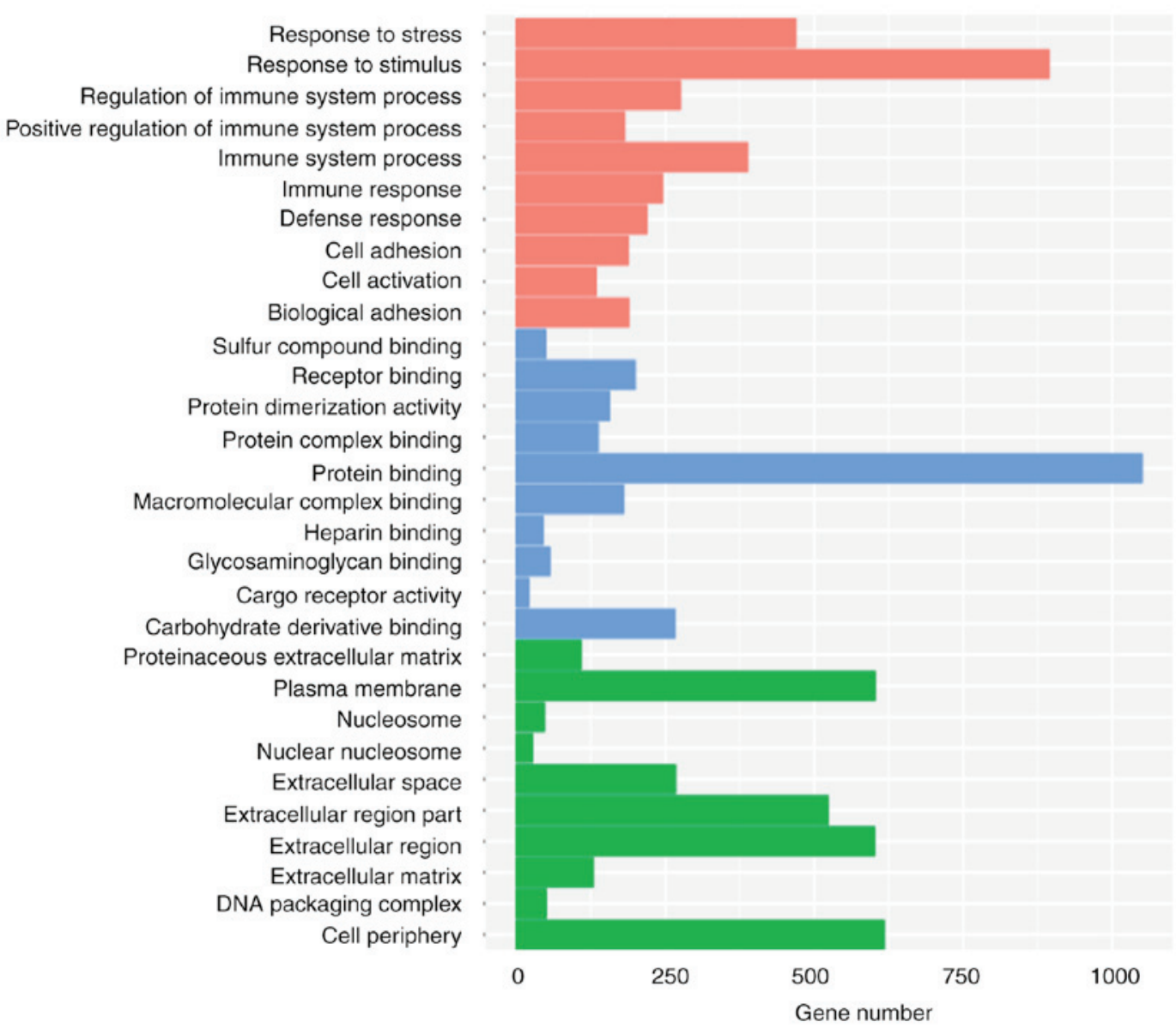

Figure 2. Top enriched GO categories in facet joint osteoarthritis. The ten most enriched GO biological processes, molecular functions and cellular components. Red represents GO biological processes, blue represents GO molecular functions and green represents GO cellular components. GO, gene ontology.

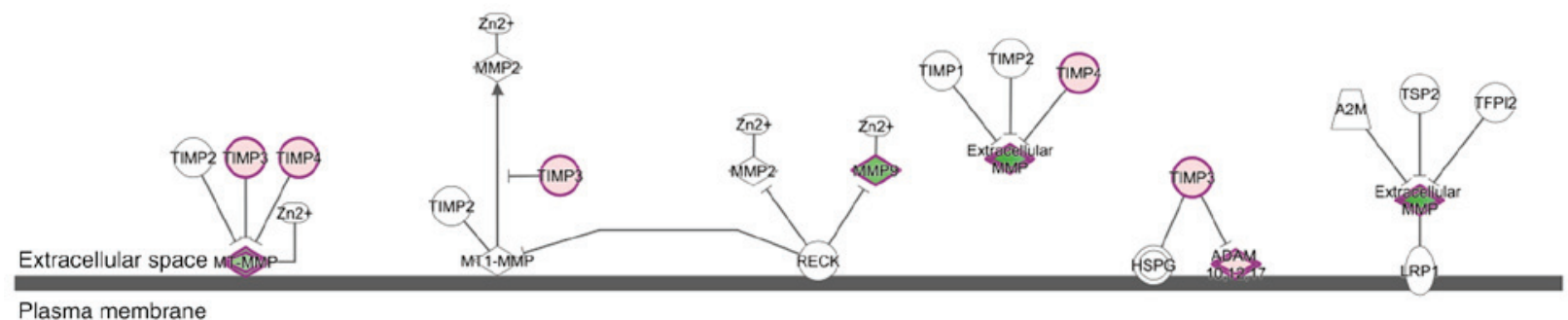

Figure 3. Schematic diagram of the canonical signaling pathway 'inhibition of matrix metalloproteinases'. Differentially expressed genes in the canonical signaling pathway 'inhibition of matrix metalloproteinases' are represented in red and green. Red indicates upregulated genes while green indicates downregulated genes. TIMP, tissue inhibitor of metalloproteinases; MMP, matrix metalloproteinase; RECK, reversion-inducing cysteine-rich protein with Kazal motifs; HSPG, basement membrane-specific heparan sulfate proteoglycan core protein; ADAM, disintegrin and metalloproteinase domain-containing protein; TSP2, thrombospondin-2; A2M, $\alpha$-2-macroglobulin; TFPI2, tissue factor pathway inhibitor 2; LRP1, prolow-density lipoprotein receptor-related protein 1; MT1-MMP, matrix metallopeptidase 14 (membrane-inserted).

Meanwhile, the expression levels of another group of metalloproteinases, ADAM proteins, were upregulated in FJOA. Similar to MMPs, ADAM proteins are also key modulators of the extracellular matrix $(40,41)$. Upregulated ADAM expression might execute opposite functions to downregulated MMPs in the breakdown and remodeling of the extracellular matrix by shedding transmembrane ligands of the epidermal growth factor (EGF) receptor and thus stimulate EGF receptor signaling. ADAM12 exhibits elevated expression levels in synovitis and post-inflammatory fibrosis of the synovial membrane in patients with early radiographic osteoarthritis (42). Polymorphisms of ADAM12, especially ADAM12 polymorphism rs3740199, are highly associated with knee osteoarthritis $(43,44)$. These previous studies indicated the involvement of ADAM12 in osteoarthritis and proposed the potential roles of ADAM in FJOA. Therefore, changes in ADAM expression and corresponding alterations in the extracellular matrix in FJOA also need to be further examined.

Overall, transcriptome sequencing and bioinformatics analysis determined the genetic changes of MMP-related genes 


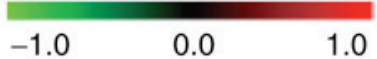

CTRL

FJOA

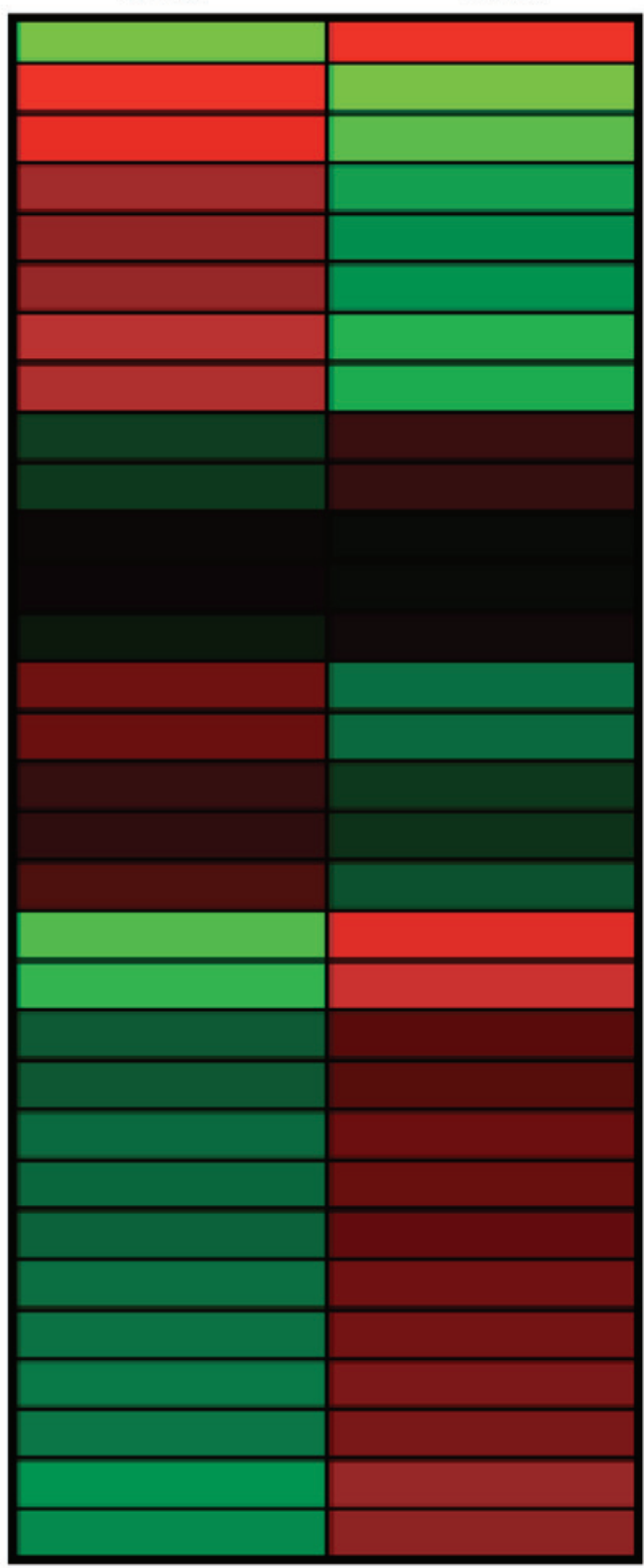

MMP23B

MMP12

MMP9

MMP3

MMP16

MMP7

MMP8

MMP25

LRP1

MMP17

A2M

ADAM17

MMP24

MMP13

MMP1

ADAM 10

TFPI2

TIMP1

ADAM12

TIMP3

MMP11

MMP15

MMP2

MMP14

RECK

HSPG2

MMP19

MMP28

TIMP2

TIMP4

TSP2

Figure 4. Heatmap of differentially expressed genes in the canonica signaling pathway 'inhibition of matrix metalloproteinases'. Red indicates upregulated genes while green indicates downregulated genes. CTRL, contro group; FJOA, facet joint osteoarthritis; TIMP, tissue inhibitor of metalloproteinases; MMP, matrix metalloproteinase; RECK, reversion-inducing cysteine-rich protein with Kazal motifs; HSPG, basement membrane-specific heparan sulfate proteoglycan core protein; ADAM, disintegrin and metalloproteinase domain-containing protein; TSP2, thrombospondin-2; A2M, $\alpha$-2-macroglobulin; TFPI2, tissue factor pathway inhibitor 2; LRP1, prolow-density lipoprotein receptor-related protein 1.

in FJOA and demonstrated the implications of the extracellular matrix and MMPs in FJOA from a molecular aspect. The present results revealed the roles of the extracellular matrix and MMPs in FJOA, contributing to the understanding of the underlying mechanisms behind FJOA. This might contribute to the investigation of novel therapeutic targets for FJOA treatment.

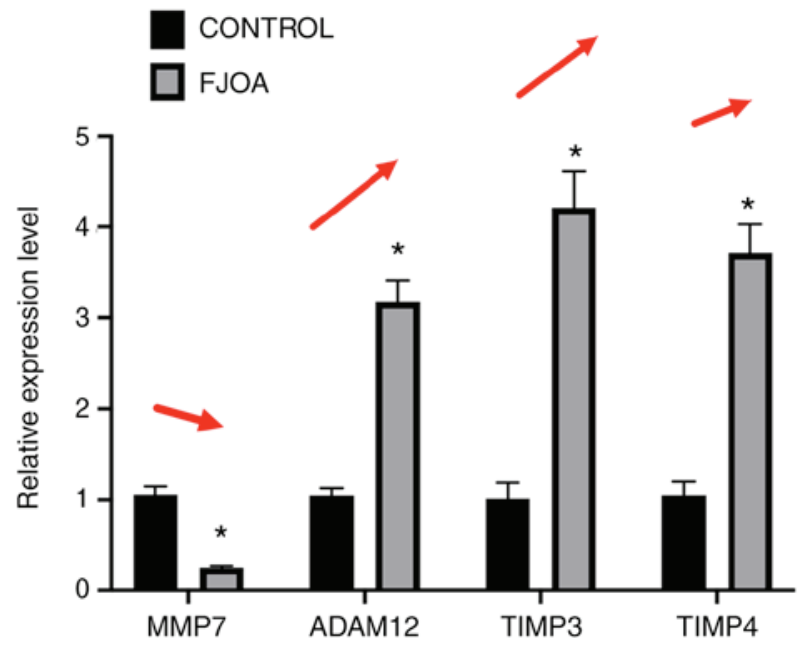

Figure 5. Reverse transcription-quantitative PCR analysis of differentially expressed genes in the canonical signaling pathway "inhibition of matrix metalloproteinases'. The relative expression levels of MMP7, ADAM12, TIMP3 and TIMP4 were calculated. The red arrows indicate the expression trends of genes, as revealed by sequencing. Data were summarized from three independent experiments. ${ }^{*} \mathrm{P}<0.05$ vs. control. FJOA, facet joint osteoarthritis; MMP7, matrix metalloproteinase 7; ADAM12, disintegrin and metalloproteinase domain-containing protein 12; TIMP, tissue inhibitor of metalloproteinases.

\section{Acknowledgements}

Not applicable.

\section{Funding}

This work was supported by supported by the Jiangsu Provincial Scientific Research Projects of '333 Project' (grant no. BRA2017204) and the Jiangsu Provincial Youth Medical Talents Subsidy Project (grant no. QNRC2016413).

\section{Availability of data and materials}

The datasets used and/or analyzed during the current study are available from the corresponding author on reasonable request.

\section{Authors' contributions}

ZC conceived and designed the experiments. CC and GX performed the experiments. CC, GX, ZC and YS analyzed the data. CC and ZC wrote the manuscript. All authors read and approved the final manuscript.

\section{Ethics approval and consent to participate}

The present study was ethically approved by the Human Ethics Committee of the Second Affiliated Hospital of Nantong University. All participants provided written informed consent.

\section{Patient consent for publication}

Not applicable. 


\section{Competing interests}

The authors declare that they have no competing interests.

\section{References}

1. Schroeppel JP, Crist JD, Anderson HC and Wang J: Molecular regulation of articular chondrocyte function and its significance in osteoarthritis. Histol Histopathol 26: 377-394, 2011.

2. García-Carbajal ZY, Garciadiego-Cázares D, Parra-Cid C, Aguilar-Gaytan R, Velasquillo C, Ibarra C and Castro Carmona JS : Cartilage tissue engineering: The role of extracellular matrix (ECM) and novel strategies. 2013 DOI: 10.5772/55917.

3. Sophia Fox AJ, Bedi A and Rodeo SA: The basic science of articular cartilage: Structure, composition, and function. Sports Health 1: 461-468, 2009.

4. Newman AP: Articular cartilage repair. Am J Sports Med 26 309-324, 1998

5. Goldring MB and Goldring SR: Osteoarthritis. J Cell Physiol 213 626-634, 2007.

6. Martel-Pelletier J, Boileau C, Pelletier JP and Roughley PJ: Cartilage in normal and osteoarthritis conditions. Best Pract Res Clin Rheumatol 22: 351-384, 2008.

7. Kim SH, Turnbull $\mathrm{J}$ and Guimond S: Extracellular matrix and cell signalling: The dynamic cooperation of integrin, proteoglycan and growth factor receptor. J Endocrinol 209: 139-1351, 2011.

8. Maldonado $\mathrm{M}$ and Nam $\mathrm{J}$ : The role of changes in extracellular matrix of cartilage in the presence of inflammation on the pathology of osteoarthritis. Biomed Res Int 2013: 284873 , 2013.

9. Prasadam I, Farnaghi S, Feng JQ, Gu W, Perry S, Crawford R and Xiao Y: Impact of extracellular matrix derived from osteoarthritis subchondral bone osteoblasts on osteocytes: Role of integrinbetal and focal adhesion kinase signaling cues. Arthritis Res Ther 15: R150, 2013.

10. Kapoor C, Vaidya S, Wadhwan V; Hitesh, Kaur G and Pathak A: Seesaw of matrix metalloproteinases (MMPs). J Cancer Res Ther 12: 28-35, 2016.

11. Steffensen B, Häkkinen L and Larjava H: Proteolytic events of wound-healing-coordinated interactions among matrix metalloproteinases (MMPs), integrins, and extracellular matrix molecules. Crit Rev Oral Biol Med 12: 373-398, 2001.

12. Jiang J, Zhang J, Wu C, Guo X, Chen C, Bao G, Sun Y, Chen J, Xue P, Xu G and Cui Z: Up-regulation of TRAF2 inhibits chondrocytes apoptosis in lumbar facet joint osteoarthritis. Biochem Biophys Res Commun 503: 1659-1665, 2018.

13. Gellhorn AC, Katz JN and Suri P: Osteoarthritis of the spine: The facet joints. Nat Rev Rheumatol 9: 216-224, 2013.

14. Nakamura A, Rampersaud YR, Sharma A, Lewis SJ Wu B, Datta P, Sundararajan K, Endisha H, Rossomacha E, Rockel JS, et al: Identification of microRNA-181a-5p and microRNA-4454 as mediators of facet cartilage degeneration JCI Insight 1: e86820, 2016.

15. Chen C, Bao GF, Xu G, Sun Y and Cui ZM: Altered Wnt and NF- $\kappa B$ Signaling in Facet Joint Osteoarthritis: Insights from RNA deep sequencing. Tohoku J Exp Med 245: 69-77, 2018.

16. Kettler A and Wilke HJ: Review of existing grading systems for cervical or lumbar disc and facet joint degeneration. Eur Spine J 15: 705-718, 2006

17. Kim D, Pertea G, Trapnell C, Pimentel H, Kelley R and Salzberg SL: TopHat2: Accurate alignment of transcriptomes in the presence of insertions, deletions and gene fusions. Genome Biol 14: R36, 2013.

18. Mortazavi A, Williams BA, McCue K, Schaeffer L and Wold B: Mapping and quantifying mammalian transcriptomes by RNA-Seq. Nat Methods 5: 621-628, 2008.

19. Huang da W, Sherman BT and Lempicki RA: Bioinformatics enrichment tools: Paths toward the comprehensive functional analysis of large gene lists. Nucleic Acids Res 37: 1-13, 2009.

20. Huang da W, Sherman BT and Lempicki RA: Systematic and integrative analysis of large gene lists using DAVID bioinformatics resources. Nat Protoc 4: 44-57, 2009.

21. Huang DW, Sherman BT, Tan Q, Kir J, Liu D, Bryant D, Guo Y, Stephens R, Baseler MW, Lane HC and Lempicki RA: DAVID bioinformatics resources: Expanded annotation database and novel algorithms to better extract biology from large gene lists. Nucleic Acids Res 35: W169-W175, 2007.
22. Livak KJ and Schmittgen TD: Analysis of relative gene expression data using real-time quantitative PCR and the 2(-Delta Delta C(T)) method. Methods 25: 402-408, 2001.

23. Reich M, Liefeld T, Gould J, Lerner J, Tamayo P and Mesirov JP: GenePattern 2.0. Nat Genet 38: 500-501, 2006.

24. Verma RP and Hansch C: Matrix metalloproteinases (MMPs): Chemical-biological functions and (Q)SARs. Bioorg Med Chem 15: 2223-2268, 2007.

25. Sternlicht MD and Werb Z: How matrix metalloproteinases regulate cell behavior. Annu Rev Cell Dev Biol 17: 463-516, 2001

26. Wasilewska A, Taranta-Janusz K, Zoch-Zwierz W, Rybi-Szumińska A and Kolodziejczyk Z: Role of matrix metalloproteinases (MMP) and their tissue inhibitors (TIMP) in nephrology. Przegl Lek 66: 485-490, 2009 (In Polish).

27. White JM: ADAMs: Modulators of Cell-cell and cell-matrix interactions. Curr Opin Cell Biol 15: 598-606, 2003.

28. Theocharis AD, Skandalis SS, Gialeli C and Karamanos NK: Extracellular matrix structure. Adv Drug Deliv Rev 97: 4-27, 2016.

29. Bonnans C, Chou J and Werb Z: Remodelling the extracellular matrix in development and disease. Nat Rev Mol Cell Biol 15: 786-801, 2014.

30. Rozario T and DeSimone DW: The extracellular matrix in development and morphogenesis: A dynamic view. Dev Biol 341: 126-140, 2010.

31. Engler AJ, Sen S, Sweeney HL and Discher DE: Matrix elasticity directs stem cell lineage specification. Cell 126: 677-689, 2006.

32. Rohani MG and Parks WC: Matrix remodeling by MMPs during wound repair. Matrix Biol 44-46: 113-121, 2015.

33. Apte SS and Parks WC: Metalloproteinases: A parade of functions in matrix biology and an outlook for the future. Matrix Biol 44-46: 1-6, 2015.

34. Bertrand J, Cromme C, Umlauf D, Frank S and Pap T: Molecular mechanisms of cartilage remodelling in osteoarthritis. Int J Biochem Cell Biol 42: 1594-1601, 2010.

35. Arpino V, Brock M and Gill SE: The role of TIMPs in regulation of extracellular matrix proteolysis. Matrix Biol 44-46: 247-254, 2015.

36. Zeng GQ, Chen AB, Li W, Song JH and Gao CY: High MMP-1, MMP-2, and MMP-9 protein levels in osteoarthritis. Genet Mol Res 14: 14811-14822, 2015.

37. Lipari L and Gerbino A: Expression of gelatinases (MMP-2, MMP-9) in human articular cartilage. Int J Immunopathol Pharmacol 26: 817-823, 2013

38. Lim NH, Meinjohanns E, Meldal M, Bou-Gharios G and Nagase H: In vivo imaging of MMP-13 activity in the murine destabilised medial meniscus surgical model of osteoarthritis. Osteoarthritis Cartilage 22: 862-868, 2014.

39. Kim JS, Ali MH, Wydra F, Li X, Hamilton JL, An HS, Cs-Szabo G, Andrews S, Moric M, Xiao G, et al: Characterization of degenerative human facet joints and facet joint capsular tissues. Osteoarthritis Cartilage 23: 2242-2251, 2015.

40. Lu P, Takai K, Weaver VM and Werb Z: Extracellular matrix degradation and remodeling in development and disease. Cold Spring Harb Perspect Biol 3: pii: a005058, 2011.

41. Wolfsberg TG, Straight PD, Gerena RL, Huovila AP, Primakoff P, Myles DG and White JM: ADAM, a widely distributed and developmentally regulated gene family encoding membrane proteins with a disintegrin and metalloprotease domain. Dev Biol 169: 378-383, 1995.

42. Kerna I, Kisand K, Suutre S, Murde M, Tamm A, Kumm J and Tamm A: The ADAM12 is upregulated in synovitis and postinflammatory fibrosis of the synovial membrane in patients with early radiographic osteoarthritis. Joint Bone Spine 81: 51-16, 2014.

43. Lv ZT, Liang S, Huang XJ, Cheng P, Zhu WT and Chen AM: Association between ADAM12 Single-nucleotide polymorphisms and knee osteoarthritis: A Meta-analysis. Biomed Res Int 2017: 5398181, 2017

44. Poonpet T, Tammachote R, Tammachote N, Kanitnate $\mathrm{S}$ and Honsawek S: Association between ADAM12 polymorphism and knee osteoarthritis in Thai population. Knee 23: 357-361, 2016.

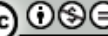

This work is licensed under a Creative Commons Attribution-NonCommercial-NoDerivatives 4.0 International (CC BY-NC-ND 4.0) License. 